\title{
Progress and puzzles in nonlinear elasticity
}

\author{
J.M. Ball \\ Oxford Centre for Nonlinear PDE, \\ Mathematical Institute, University of Oxford, \\ 24-29 St. Giles', Oxford OX1 3LB, U.K.
}

\section{Introduction}

These lectures are largely based on two previous survey articles [6], [7], and cover a selection of open problems with some new remarks and updates. But they also give an introduction to the convexity conditions that are the objects of study of this course.

We begin by considering the usual set-up for nonlinear elastostatics, in which an elastic body occupies in a reference configuration the bounded domain (i.e. open and connected set) $\Omega \subset \mathbf{R}^{3}$ having Lipschitz boundary $\partial \Omega$. We assume that the boundary can be decomposed as $\partial \Omega=\partial \Omega_{1} \cup \partial \Omega_{2} \cup$ $N$, where $\partial \Omega_{1}, \partial \Omega_{2}$ are relatively open and disjoint, and where $N$ has zero area (that is, its two-dimensional Hausdorff measure $\mathcal{H}^{2}(N)=0$ ).

For a deformation $\mathbf{y}: \Omega \rightarrow \mathbf{R}^{3}$, the deformation gradient

$$
D \mathbf{y}(\mathbf{x})=\left(\frac{\partial y_{i}(x)}{\partial x_{j}}\right)
$$

is required to belong to $M_{+}^{3 \times 3}$, where $M^{m \times n}=\{$ real $m \times n$ matrices $\}$, and $M_{+}^{n \times n}=\left\{\mathbf{A} \in M^{n \times n}: \operatorname{det} \mathbf{A}>0\right\}$. We suppose that $\mathbf{y}$ satisfies mixed displacement zero-traction boundary conditions, so that

$$
\left.\mathbf{y}\right|_{\partial \Omega_{1}}=\overline{\mathbf{y}}(\cdot),
$$

where $\overline{\mathbf{y}}: \partial \Omega_{1} \rightarrow \mathbf{R}^{3}$ is given.

We further assume that the body is comprised of homogeneous material, that is the material response is the same at each point $\mathbf{x} \in \Omega$. (Note that this is not the same as having the same material at each point; think, for example, of two elastic bands stuck together, one stretched relative to the 
other, so that there is no stress-free reference configuration.) We also assume that the temperature is constant. The total elastic energy is then given by

$$
I(\mathbf{y})=\int_{\Omega} W(D \mathbf{y}(\mathbf{x})) d \mathbf{x}
$$

where the stored-energy function $W: M_{+}^{3 \times 3} \rightarrow \mathbf{R}$ is assumed to be $C^{1}$ and bounded below (in fact we may and do assume that $W \geq 0$ ). The Piola-Kirchhoff stress tensor is then given by $\mathbf{T}_{R}(\mathbf{A})=D_{\mathbf{A}} W(\mathbf{A})$.

Let $\varphi: \Omega \rightarrow \mathbf{R}^{3}$ be smooth with $\left.\varphi\right|_{\partial \Omega_{1}}=0$. Formally computing

$$
\left.\frac{d}{d \tau} I(\mathbf{y}+\tau \varphi)\right|_{\tau=0}=0
$$

we obtain the weak form of the Euler-Lagrange equation

$$
\int_{\Omega} D_{\mathbf{A}} W(D \mathbf{y}) \cdot D \varphi d \mathbf{x}=0 \quad \text { for all such } \varphi . \quad(\text { WEL })
$$

If $\mathbf{y}, \partial \Omega_{1}, \partial \Omega_{2}$ are sufficiently regular then (WEL) is equivalent to

$$
\left.\begin{array}{ll}
\operatorname{Div} D_{\mathbf{A}} W(D \mathbf{y}(\mathbf{x})) & =0 \text { for } \mathbf{x} \in \Omega, \\
D_{\mathbf{A}} W(D \mathbf{y}(\mathbf{x})) \mathbf{N}(\mathbf{x}) & =0 \text { for } \mathbf{x} \in \partial \Omega_{2},
\end{array}\right\}
$$

where $\mathbf{N}(\mathbf{x})$ is the unit outward normal to $\partial \Omega_{2}$. (Thus the zero traction boundary condition on $\partial \Omega_{2}$ appears as a natural boundary condition.)

\subsection{Function Spaces}

To what function space should $\mathbf{y}$ belong? This is part of the mathematical model, since examples show that the minimum (or infimum) of $I$ in different function spaces can be different. We will assume that $\mathbf{y}$ belongs to the (largest) Sobolev space $W^{1,1}=W^{1,1}\left(\Omega, \mathbf{R}^{3}\right)$, where for $1 \leq p \leq \infty$

$$
\begin{aligned}
W^{1, p}\left(\Omega ; \mathbf{R}^{3}\right) & =\left\{\mathbf{z}: \Omega \rightarrow \mathbf{R}^{3},\|\mathbf{z}\|_{1, p}<\infty\right\} \\
\|\mathbf{z}\|_{1, p} & =\left(\int_{\Omega}\left(|\mathbf{z}|^{p}+|D \mathbf{z}|^{p}\right) d \mathbf{x}\right)^{\frac{1}{p}} \text { if } 1 \leq p<\infty \\
& =\underset{\mathbf{x} \in \Omega}{\operatorname{ess~sup}}(|\mathbf{z}(\mathbf{x})|+|D \mathbf{z}(\mathbf{x})|) \text { if } p=\infty .
\end{aligned}
$$

(For the formal definitions and basic facts see standard texts on Sobolev spaces e.g. Adams \& Fournier [2].)

If $\mathbf{y} \in W^{1,1}$ then $\mathbf{y}$ is absolutely continuous along a.e. line parallel to the coordinate axes (see Morrey [27]). Hence deformations with planar cracks 
are excluded, though discontinuities on sets $S(\mathbf{y}) \subset \Omega$ with $\mathcal{H}^{2}(S(\mathbf{y}))=0$ may be possible, as for example in cavitation (see [5] and Section 1.4 below). How should we decide on the 'correct' function space? We could hope to do this by means of a passage from an atomistic/molecular model to a continuum one. Such a 'derivation' of the continuum model would certainly lead to a larger function space than $W^{1,1}$ (allowing fracture, for example) and a modified energy functional. It would then be a task to understand the status of minimizers of $I$ in $W^{1,1}$ with respect to the modified theory (e.g. as metastable states).

\subsection{Properties of $W$}

We make the following hypotheses on $W$. The first is frame-indifference:

$$
W(\mathbf{R A})=W(\mathbf{A}) \text { for all } \mathbf{R} \in S O(3), \mathbf{A} \in M_{+}^{3 \times 3},
$$

where $S O(3)=\left\{\mathbf{R} \in M_{+}^{3 \times 3}: \mathbf{R}^{T} \mathbf{R}=\mathbf{1}\right\}$, which holds if and only if

$$
W(\mathbf{A})=W(\mathbf{U}), \quad \mathbf{U}=\left(\mathbf{A}^{T} \mathbf{A}\right)^{\frac{1}{2}} .
$$

The second is material symmetry:

$$
W(\mathbf{A Q})=W(\mathbf{A}) \text { for all } \mathbf{Q} \in \mathcal{S}, \mathbf{A} \in M_{+}^{3 \times 3},
$$

where $\mathcal{S}$ is the isotropy group of the material. The case $\mathcal{S} \supset S O(3)$ corresponds to an isotropic material, for which we have the representation

$$
W(\mathbf{A})=\Phi\left(v_{1}, v_{2}, v_{3}\right),
$$

where the $v_{i}$ are the principal stretches (that is, the eigenvalues of $\mathbf{U}$ ) and $\Phi$ is symmetric with respect to permutations of the $v_{i}$.

The third condition says that infinite energy is required to compress the material to zero volume:

$$
W(\mathbf{A}) \rightarrow \infty \text { as } \operatorname{det} \mathbf{A} \rightarrow 0+.
$$

We set $W(\mathbf{A})=\infty$ if $\operatorname{det} \mathbf{A} \leq 0$. Then $I(\mathbf{y}) \in[0, \infty]$ is well defined for $\mathbf{y} \in W^{1,1}$, and if $I(\mathbf{y})<\infty$ then $\operatorname{det} D \mathbf{y}(\mathbf{x})>0$ a.e..

Are there any other conditions on $W$ satisfied by 'all materials'? In the older literature there was a feeling that there should be such 'constitutive inequalities' that would correspond to 'stress increasing with strain' (for a comprehensive discussion see Truesdell \& Noll [34]). Two such candidates were the strong ellipticity condition and the Coleman-Noll inequality. The strong ellipticity condition is

$$
D^{2} W(\mathbf{A})(\mathbf{a} \otimes \mathbf{N}, \mathbf{a} \otimes \mathbf{N})>0, \quad \text { for all } \mathbf{a}, \mathbf{N} \in \mathbf{R}^{3},|\mathbf{N}|=1 .
$$


where $(\mathbf{a} \otimes \mathbf{N})_{i \alpha}=a_{i} N_{\alpha}$, that is

$$
\left.\frac{d^{2}}{d t^{2}} W(\mathbf{A}+t \mathbf{a} \otimes \mathbf{N})\right|_{t=0}=\frac{\partial^{2} W(\mathbf{A})}{\partial A_{i \alpha} \partial A_{j \beta}} a_{i} N_{\alpha} a_{j} N_{\beta}>0 .
$$

In particular this condition implies the reality of wave speeds in elastodynamics.

We do not state the Coleman-Noll inequality here, but note that for an isotropic material it implies that $\Phi\left(v_{1}, v_{2}, v_{3}\right)$ is convex. It is easily seen that this is not satisfied for rubber because rubber is almost incompressible. For example, for moderately large $v$ the convexity inequality

$$
\Phi\left(\frac{1}{2}\left(v+v^{-1}\right), \frac{1}{2}\left(v+v^{-1}\right), 1\right) \leq \frac{1}{2}\left(\Phi\left(v, v^{-1}, 1\right)+\Phi\left(v^{-1}, v, 1\right)\right)
$$

is not satisfied because the volume change $\frac{1}{4}\left(v+v^{-1}\right)^{2}$ is large and thus the left-hand side large compared to the right-hand side. Thus the ColemanNoll inequality is not generally satisfied.

In fact 'stress increases with strain' should be regarded as a stability condition. For example, in one dimension consider the minimizers $y$ of

$$
I(y)=\int_{0}^{1} W\left(y_{x}\right) d x, \text { subject to } y(0)=0, y(1)=\lambda>0,
$$

where $y_{x}=d y / d x$. Suppose $W \in C^{1}(0, \infty), W(p) \rightarrow \infty$ as $p \rightarrow 0+$, $\lim _{p \rightarrow 0+} \frac{W(p)}{p}=\infty$. Let $W^{* *}$ be the convexification of $W$ (that is the greatest convex function less than or equal to $W$ ). It is not difficult to show that $W^{* *}$ is $C^{1}$ (for a general result of this type see Kirchheim \& Kristensen [20]). A Weierstrass point $p$ is a point at which $W(p)=W^{* *}(p)$, so that the tangent at $p$ to the graph of $W$ does not lie above the graph. Let

$$
I^{* *}(y)=\int_{0}^{1} W^{* *}\left(y_{x}\right) d x .
$$

We can think of $W^{* *}$ as being the macroscopic stored-energy function corresponding to the mesoscopic stored-energy function $W$. In fact, setting $\mathcal{A}=\left\{y \in W^{1,1}(0,1): y(0)=0, y(1)=\lambda, y_{x}>0\right.$ a.e. $\}$ we have that

$$
\inf _{\mathbf{y} \in \mathcal{A}} I(y) \geq \inf _{y \in \mathcal{A}} I^{* *}(y) \geq \inf _{y \in \mathcal{A}} W^{* *}\left(\int_{0}^{1} y_{x} d x\right)=W^{* *}(\lambda),
$$

where the middle inequality follows from Jensen's inequality. But any $\lambda>0$ can be written as $\lambda=\mu p+(1-\mu) q$, where $W(p)=W^{* *}(p), W(q)=$ 
$W^{* *}(q), 0 \leq \mu \leq 1$, where $0<p \leq \lambda \leq q<\infty$ and $W^{* *}(r)$ is affine for $r \in[p, q]$. Thus

$$
y^{\lambda}(x)= \begin{cases}p x, & 0 \leq x \leq \mu \\ p \mu+q(x-\mu), & \mu \leq x \leq 1\end{cases}
$$

is such that

$$
I\left(y^{\lambda}\right)=\mu W^{* *}(p)+(1-\mu) W^{* *}(q)=W^{* *}(\lambda) .
$$

Hence $y^{\lambda}$ is a minimizer, and $\inf _{\mathcal{A}} I=\inf _{\mathcal{A}} I^{* *}$. For any minimizer $y^{*}$ we have $W\left(y_{x}^{*}\right)=W^{* *}\left(y_{x}^{*}\right)$ a.e., so that the only values of $y_{x}^{*}$ that can be observed in minimizers (in fact even in strong local minimizers, i.e. local minimizers in the $C^{0}$ metric) are Weierstrass points. Also we have that $W_{p}\left(y_{x}^{*}\right)=W_{p}^{* *}(\lambda)$ a.e., so that the stress is constant and a monotone function of the overall strain $\lambda$, even though no assumption has been made about monotonicity of $W_{p}(p)=d W(p) / p$ in $p$ (i.e. of convexity of $W$ ).

In higher dimensions the role played in one dimension by convexity is played by quasiconvexity (in the sense of Morrey [26]). Let $f: M^{m \times n} \rightarrow$ $\mathbf{R} \cup\{+\infty\}$ be Borel measurable and bounded below. We say that $f$ is quasiconvex at $\mathbf{A} \in M^{m \times n}$ if

$$
\int_{\Omega} f(\mathbf{A}+D \varphi(\mathbf{x})) d \mathbf{x} \geq \int_{\Omega} f(\mathbf{A}) d \mathbf{x}
$$

for any $\varphi \in C_{0}^{\infty}\left(\Omega ; \mathbf{R}^{m}\right)$, and is quasiconvex if it is quasiconvex at every $\mathbf{A} \in M^{m \times n}$. Here $\Omega \subset \mathbf{R}^{n}$ is any bounded open set whose boundary $\partial \Omega$ has zero $n$-dimensional Lebesgue measure. A standard scaling argument shows that these definitions do not depend on $\Omega$.

\subsection{Roles of quasiconvexity in the calculus of variations}

Existence of global minimizers (Morrey [26; 27], Acerbi \& Fusco [1])

If $f: M^{m \times n} \rightarrow \mathbf{R}$ is quasiconvex and satisfies

$$
C_{1}|\mathbf{A}|^{p}-C_{0} \leq f(\mathbf{A}) \leq C_{2}\left(|\mathbf{A}|^{p}+1\right) \text { for all } \mathbf{A} \in M^{m \times n},
$$

where $p>1, C_{0}$ and $C_{1}>0, C_{2}>0$ are constants, then

$$
\mathcal{F}(\mathbf{y})=\int_{\Omega} f(D \mathbf{y}) d \mathbf{x}
$$

attains a global minimum on

$$
\mathcal{A}=\left\{\mathbf{y} \in W^{1,1}\left(\Omega ; \mathbf{R}^{m}\right):\left.\mathbf{y}\right|_{\partial \Omega_{1}}=\overline{\mathbf{y}}\right\} .
$$


Here we assume that $\Omega$ has Lipschitz boundary $\partial \Omega, \partial \Omega_{1} \subset \partial \Omega$ is $\mathcal{H}^{n-1}$ measurable, and $\overline{\mathbf{y}}: \partial \Omega_{1} \rightarrow \mathbf{R}^{m}$ is given such that $\mathcal{A}$ is nonempty. The proof is by the direct method of the calculus of variations, using the fact that under the growth conditions (3) quasiconvexity is necessary and sufficient for $\mathcal{F}$ to be sequentially weakly lower semicontinuous on $W^{1, p}\left(\Omega ; \mathbf{R}^{m}\right)$. A result of Ball \& Murat [11] shows that if the minimum of $\mathcal{F}$ is attained whenever suitable lower order terms $g(\mathbf{y})$ are added to the integrand, then $W$ is quasiconvex. This shows that the direct method is the right method for proving existence.

Relaxation (Dacorogna [13])

Under similar hypotheses we have

$$
\inf _{\mathcal{A}} \mathcal{F}=\inf _{\mathcal{A}} \mathcal{F}^{q c}
$$

where

$$
\mathcal{F}^{q c}(\mathbf{y})=\int_{\Omega} f^{q c}(D \mathbf{y}) d x
$$

and $f^{q c}$ is the quasiconvex envelope of $f$, i.e the supremum of all quasiconvex functions $g \leq f$. In elasticity this has the interpretation that for problems (such as elastic crystals) for which the total elastic energy $I$ does not attain a minimum, the macroscopic stored-energy function corresponding to the microscopic/mesoscopic stored-energy function $W$ is $W^{q c}$. In Ball, Kirchheim \& Kristensen [8] it is shown that if $f \in C^{1}$ then $f^{q c}$ is $C^{1}$.

Partial regularity of energy minimizers (Evans [15], Kristensen \& Taheri [24])

Under similar hypotheses, with a slightly strengthened version of quasiconvexity, and assuming $f$ smooth, any global (or $W^{1, p}$-local) minimizer is smooth on the complement of a closed set $E$ of $n$-dimensional Lebesgue measure zero. For Lipschitz minimizers the Hausdorff dimension of the singular set is strictly less than $n$ (see Kristensen \& Mingione [23]).

\section{Necessary and sufficient conditions for local minimizers}

Again consider

$$
\mathcal{F}(\mathbf{y})=\int_{\Omega} f(D \mathbf{y}) d \mathbf{x},
$$

where $f \in C^{2}$ is bounded below, and suppose that $\mathbf{y} \in \mathcal{A} \cap C^{1}\left(\bar{\Omega} ; \mathbf{R}^{m}\right)$ is a $W^{1, p}$ local minimizer of $\mathcal{F}$ in $\mathcal{A}$, i.e. for some $\varepsilon>0$ we have that

$$
\mathcal{F}(\mathbf{z}) \geq \mathcal{F}(\mathbf{y}) \text { for all } z \in \mathcal{A} \text { with }\|\mathbf{z}-\mathbf{y}\|_{1, p}<\varepsilon .
$$


Then

(NC1) y satisfies (WEL):

$$
\int_{\Omega} D f(D \mathbf{y}) \cdot D \varphi d \mathbf{x}=0 \text { for all smooth } \varphi \text { with }\left.\varphi\right|_{\partial \Omega_{1}}=0 .
$$

(NC2) (Positivity of the second variation)

For such $\varphi$

$$
\left.\frac{d^{2}}{d \tau^{2}} \mathcal{F}(\mathbf{y}+\tau \varphi)\right|_{\tau=0} \geq 0
$$

that is

$$
\int_{\Omega} D_{\mathbf{A}}^{2} f(D \mathbf{y})(D \varphi, D \varphi) d \mathbf{x} \geq 0 \text { for all smooth } \varphi \text { with }\left.\varphi\right|_{\partial \Omega_{1}}=0 .
$$

(NC3) (Interior quasiconvexity)

If $\mathbf{x}_{0} \in \Omega$ then $f$ is quasiconvex at $D \mathbf{y}\left(\mathbf{x}_{0}\right)$.

(NC4) (Quasiconvexity at the free boundary) (Ball \& Marsden [9])

Let $\mathbf{x}_{1} \in \partial \Omega_{2}=\partial \Omega \backslash \partial \Omega_{1}$. We assume that $\partial \Omega$ is $C^{1}$ with unit outward normal $\mathbf{N}\left(\mathbf{x}_{1}\right)$ at $\mathbf{x}_{1}$. Let $B^{-}\left(\mathbf{x}_{1}\right)$ be the half-ball $\left\{\mathbf{x} \in \mathbf{R}^{n}:|\mathbf{x}|<1, \mathbf{x}\right.$. $\left.\mathbf{N}\left(\mathbf{x}_{1}\right)<0\right\}$. Then

$$
\int_{B^{-}\left(\mathbf{x}_{1}\right)} f\left(D \mathbf{y}\left(\mathbf{x}_{1}\right)+D \varphi(\mathbf{z})\right) d \mathbf{z} \geq \int_{B^{-}\left(\mathbf{x}_{1}\right)} f\left(D \mathbf{y}\left(\mathbf{x}_{1}\right)\right) d \mathbf{z}
$$

whenever $\varphi \in C^{\infty}\left(\overline{B^{-}\left(\mathbf{x}_{1}\right)} ; \mathbf{R}^{m}\right)$ with $\left.\varphi\right|_{\partial B^{-}\left(\mathbf{x}_{1}\right) \cap \partial B(0,1)}=0$.

(NC3) and (NC4) are generalizations of the classical Weierstrass condition. It is natural to ask whether ( $\mathrm{NC} 1)-(\mathrm{NC} 4)$ can be slightly strengthened to form a set of sufficient conditions for $\mathbf{y}$ to be a strong local or $W^{1, p}$ local minimizer. For example, (NC2) can be strengthened to

$$
(\mathrm{NC} 2)^{+} \quad \int_{\Omega} D_{\mathbf{A}}^{2} f(D \mathbf{y})(D \varphi, D \varphi) d \mathbf{x} \geq \mu \int_{\Omega}|D \varphi|^{2} d \mathbf{x}
$$

for all smooth $\varphi$ with $\left.\varphi\right|_{\partial \Omega_{1}}=0$, for some $\mu>0$. This has been achieved in very interesting recent work of Grabovsky \& Mengesha [17], in the more general context of integrands $f(\mathbf{x}, \mathbf{y}, D \mathbf{y})$ satisfying suitable $p^{\text {th }}$ power growth conditions, thus generalizing the classical Weierstrass sufficiency theorem to this case. The idea is to split an arbitrary variation into a 'weak' and a 'strong' part.

Unfortunately none of these results applies directly to elasticity, since the growth conditions assumed are inconsistent with the condition (2). This is 
related to the lack of a tractable characterization of quasiconvexity, which might lead to different proof techniques. It is known (Kristensen [22]) that there is no local characterization. In general we have that for $f$ taking finite values

$$
f \text { polyconvex } \Rightarrow f \text { quasiconvex } \Rightarrow f \text { rank-one convex. }
$$

Here $f$ polyconvex means that $f(\mathbf{A})=g(\mathbf{J}(\mathbf{A}))$ for some convex function $g$ of the list $\mathbf{J}(\mathbf{A})$ of all minors of $\mathbf{A}$, while $f$ rank-one convex means that $t \mapsto f(\mathbf{A}+t \mathbf{a} \otimes \mathbf{N})$ is convex for all $\mathbf{a} \in \mathbf{R}^{m}, \mathbf{N} \in \mathbf{R}^{n}$. The converse implications are false for $m>1, n>1$ except that when $m=2$ it is not known whether $f$ rank-one convex implies $f$ quasiconvex (for $m>2$ this is the famous counterexample of Šverák [32]). Although there are examples of quasiconvex $f$ that are not polyconvex, no useful class of examples is known. Existence theorems based on polyconvexity remain of interest both because of this lack of examples and because they can handle the blow-up of $W(\mathbf{A})$ as $\operatorname{det} \mathbf{A} \rightarrow 0+$. The following such result is due to Müller, Qi \& Yan [28], following [3].

Theorem 1.1. Suppose that $W$ satisfies

(H1) $W$ is polyconvex, i.e. $W(\mathbf{A})=g(\mathbf{A}, \operatorname{cof} \mathbf{A}$, $\operatorname{det} \mathbf{A})$ for all $\mathbf{A} \in M_{+}^{3 \times 3}$ and some convex $g$,

(H2) $W(\mathbf{A}) \geq c_{0}\left(|\mathbf{A}|^{2}+|\operatorname{cof} \mathbf{A}|^{\frac{3}{2}}\right)-c_{1}$, for all $\mathbf{A} \in M_{+}^{3 \times 3}$, where $c_{0}>0$.

Then if $\mathcal{A}$ is nonempty, there exists a global minimizer $\mathbf{y}^{*}$ of $I$ in $\mathcal{A}$.

\subsection{Open problems in elastostatics}

When is the minimizer $\mathbf{y}^{*}$ smooth? No such result is known even in the simplest special cases, such as

$$
W(\mathbf{A})=|\mathbf{A}|^{2}+|\mathbf{A}|^{4}+h(\operatorname{det} \mathbf{A}),
$$

where $h$ is smooth, convex, with $h(\delta) \rightarrow \infty$ as $\delta \rightarrow 0+, \frac{h(\delta)}{\delta} \rightarrow \infty$ as $\delta \rightarrow \infty$. Although there are counterexamples to regularity for minimizers of

$$
\mathcal{F}(\mathbf{y})=\int_{\Omega} f(D \mathbf{y}) d \mathbf{x}
$$

where $f$ is strictly convex (see Nečas [29], Šverák \& Yan [33]), none are known for the dimensions $m=n=2$ or 3 .

Does $\mathrm{y}^{*}$ satisfy (WEL)? The difficulty is that (WEL) requires that $D_{A} W\left(D \mathbf{y}^{*}\right)$ be at least locally integrable, but $I\left(\mathbf{y}^{*}\right)<\infty$ only tells us that 
$W\left(D \mathbf{y}^{*}\right) \in L^{1}$, and $\left|D_{\mathbf{A}} W(\mathbf{A})\right|$ may be much bigger than $W(\mathbf{A})$ when $|\mathbf{A}|$ is large or $\operatorname{det} \mathbf{A}$ is small, so that there is no obvious way to pass to the limit $t \rightarrow 0$ in the difference quotient

$$
\int_{\Omega} \frac{W\left(D \mathbf{y}^{*}+t D \varphi\right)-W\left(D \mathbf{y}^{*}\right)}{t} d \mathbf{x}
$$

In fact it need not be the case that $\operatorname{det}\left(D \mathbf{y}^{*}(x)+D \varphi(\mathbf{x})\right)>0$.

There is no general such theorem even in the one-dimensional calculus of variations. An example (see Ball \& Mizel [10]) is the problem of minimizing

$$
I(u)=\int_{-1}^{1}\left[\left(x^{4}-u^{6}\right)^{2} u_{x}^{28}+\varepsilon u_{x}^{2}\right] d x
$$

subject to $u(-1)=-1, u(1)=1$, where $0<\varepsilon<\varepsilon_{0}<<1$, which has a global minimizer $u^{*}$ with

$$
u^{*}(x) \sim|x|^{-\frac{1}{3}} x
$$

as $x \sim 0$. In one dimension (WEL) is equivalent to the integrated form

$$
f_{u_{x}}=\int_{0}^{x} f_{u} d s+\text { const. }
$$

but here $f_{u_{x}}\left(x, u^{*}, u_{x}^{*}\right)$ is unbounded.

It is possible to derive two different forms of the Euler-Lagrange equation for (1) by taking variations that are compositions, thus preserving the sign of the determinant. For example, by considering the variation

$$
\mathbf{y}_{\tau}(\mathbf{x})=\mathbf{y}^{*}(\mathbf{x})+\tau \varphi\left(\mathbf{y}^{*}(\mathbf{x})\right)
$$

we can prove that Cauchy's equilibrium equations hold in the weak form

$$
\int_{\Omega}\left[D_{\mathbf{A}} W\left(D \mathbf{y}^{*}\right) D \mathbf{y}^{* T} \cdot D \varphi\left(\mathbf{y}^{*}\right)\right] d \mathbf{x}=0
$$

for all $\varphi \in C^{1}\left(\mathbf{R}^{3} ; \mathbf{R}^{3}\right)$ with $\varphi, D \varphi$ uniformly bounded and such that $\left.\varphi\left(\mathbf{y}^{*}\right)\right|_{\partial \Omega_{1}}=$ 0 , provided that $W$ satisfies

$$
\left|D_{\mathbf{A}} W(\mathbf{A}) A^{T}\right| \leq K(W(\mathbf{A})+1) \quad \text { for all } \mathbf{A} \in M_{+}^{3 \times 3},
$$

a condition that holds for many models of natural rubber (for the details see $[7])$. 
Prove or disprove that under suitable growth conditions on $W$, $\operatorname{det} D \mathbf{y}^{*}(\mathbf{x}) \geq \varepsilon>0$. For this we seem to need some variation that increases det $D \mathbf{y}^{*}$ where it is small. Perhaps related to this is the open problem

If $\mathbf{y} \in W^{1, p}\left(\Omega, \mathbf{R}^{3}\right)$ is invertible, can $\mathbf{y}$ be approximated in $W^{1, p}$ by piecewise affine invertible maps? The difficulty can be seen even in two dimensions, where a Lipschitz y can map three points $A, B, C$ to points $A^{\prime}, B^{\prime}, C^{\prime}$ in such a way that the orientation of the triangle $A B C$ is opposite to that of $A^{\prime} B^{\prime} C^{\prime}$. For some recent partial results see Bellido \& Mora-Corral [12], Mora-Corral [25].

If $\Omega$ is homeomorphic to a ball, $\partial \Omega=\partial \Omega_{1}, W$ strictly polyconvex, are minimizers (or sufficiently smooth equilibrium solutions) unique? There are well-known counterexamples to uniqueness when $\partial \Omega_{1} \neq$ $\partial \Omega$ or if $\Omega$ has holes (see [7] Section 2.6). The answer to the problem as stated is probably no, as explained in [7]. A recent paper by Spadaro [31] gives some counterexamples with $\mathbf{y}: \Omega \rightarrow \mathbf{R}^{3}, \Omega \subset \mathbf{R}^{2}$, with injective boundary values, using ideas from minimal surfaces. However it is not clear how to extend these examples to $\mathbf{y}: \Omega \rightarrow \mathbf{R}^{n}, n=2$ or $n=3$, where $\Omega \subset \mathbf{R}^{n}$ and the boundary values are injective.

Now consider the example

$$
W(\mathbf{A})=|\mathbf{A}|^{2}+h(\operatorname{det} \mathbf{A}),
$$

where $h$ is convex, $h(\delta) \rightarrow \infty$ as $\delta \rightarrow 0, \frac{h(\delta)}{\delta} \rightarrow \infty$ as $\delta \rightarrow \infty$. This $W$ is polyconvex, but does not satisfy the growth condition (H2). It is an example of a function that is $W^{1, p}$ quasiconvex, i.e.

$$
\int_{\Omega} W(\mathbf{A}+D \varphi(\mathbf{x})) d \mathbf{x} \geq \int_{\Omega} W(\mathbf{A}) d \mathbf{x} \quad \text { for all } \varphi \in W_{0}^{1, p}\left(\Omega ; \mathbf{R}^{3}\right)
$$

if $p \geq 3$, but not if $p<3$. In fact if $\mathbf{A}=\lambda \mathbf{1}$, with $\lambda>0$ sufficiently large, we can find a radial deformation of the form

$$
\mathbf{y}(\mathbf{x})=\frac{r(R)}{R} x
$$

with $r(0)>0, r(1)=\lambda$, and $I(\mathbf{y})<I(\lambda \mathbf{1})$. This is the phenomenon of cavitation.

For such a $W$, is the minimum of $I$ attained? Here is a strange argument, perhaps suggesting that the answer is no. Let us suppose that 
the minimum is attained for the cube $Q=(-1,1)^{3}$ and linear boundary data $\left.\mathbf{y}\right|_{\partial Q}=A \mathbf{x}$, and that the minimizer $\mathbf{y}^{*}$ is $C^{1}$ up to the flat parts of the boundary $\partial Q$. We can deduce from this that $D \mathbf{y}^{*}$ is constant on each face of the cube. To see this pick some point $\mathbf{a}$ in the interior of one face of the cube, having normal $\mathbf{e}_{1}$ say, and another point $\mathbf{b}$ in the interior of the opposite face. Now for some small $\varepsilon>0$ consider the two cubes $Q_{1}=\varepsilon Q$ and $Q_{2}=\varepsilon(Q+\mathbf{a}-\mathbf{b})$. These cubes are disjoint, interior to $Q$, and their closures meet on part of the surface $\left\{\mathbf{x}: \mathbf{x} \cdot \mathbf{e}_{1}=\varepsilon \mathbf{a} \cdot \mathbf{e}_{1}\right\}$ which has $\varepsilon \mathbf{a}$ as an interior point. Now let $\mathbf{c}_{1}=0, \mathbf{c}_{2}=\varepsilon(\mathbf{a}-\mathbf{b}), \varepsilon_{1}=\varepsilon_{2}=\varepsilon$ and choose cubes $Q_{j}=\varepsilon_{j} Q+\mathbf{c}_{j}, j \geq 3$ such that the $\left\{Q_{i}\right\}_{i=1}^{\infty}$ are disjoint with

$$
\operatorname{meas}\left(Q \backslash \bigcup_{i=1}^{\infty} Q_{i}\right)=0 \text {, }
$$

which is possible by Vitali's covering theorem. Define for $\mathbf{x} \in \bar{Q}$

$$
\mathbf{y}(\mathbf{x})= \begin{cases}A \mathbf{c}_{i}+\varepsilon \mathbf{y}^{*}\left(\frac{\mathbf{x}-\mathbf{c}_{i}}{\varepsilon}\right) & \text { if } \mathbf{x} \in Q_{i} \\ A \mathbf{x} & \text { otherwise }\end{cases}
$$

Then $\mathbf{y} \in \mathbf{A} \mathbf{x}+W_{0}^{1, p}\left(Q ; \mathbf{R}^{3}\right)$ and

$$
I(\mathbf{y})=\sum_{i=1}^{\infty} \int_{Q_{i}} W\left(D \mathbf{y}^{*}\left(\frac{\mathbf{x}-\mathbf{c}_{i}}{\varepsilon}\right)\right) d \mathbf{x}=I\left(\mathbf{y}^{*}\right) .
$$

Hence $\mathbf{y}$ is also a minimizer, and since $\mathbf{y}$ is piecewise $C^{1}$ in the neighbourhood of $\varepsilon \mathbf{a}$ it follows that in this neighbourhood it satisfies (WEL). Consequently the stress at $\varepsilon \mathbf{a}$ across the surface $\left\{\mathbf{x}: \mathbf{x} \cdot \mathbf{e}_{1}=\varepsilon \mathbf{a} \cdot \mathbf{e}_{1}\right\}$ is continuous, i.e.

$$
D W\left(D \mathbf{y}^{*}(\mathbf{a})\right) \mathbf{e}_{1}=D W\left(D \mathbf{y}^{*}(\mathbf{b})\right) \mathbf{e}_{1} .
$$

But since $W$ is strictly polyconvex it is strictly rank-one convex, and hence by a result in [4] (see also Knowles \& Sternberg [21]) we have $D \mathbf{y}^{*}(\mathbf{a})=$ $D \mathbf{y}^{*}(\mathbf{b})$. Since $\mathbf{a}$ and $\mathbf{b}$ are arbitrary points on opposite faces of $Q$ the claim follows.

Can we incorporate cavitation into a more general theory of fracture? The 'free-discontinuity' variational models of fracture (see e.g. Francfort \& Marigo [16]) are based on minimization of an energy such as

$$
I(\mathbf{y})=\int_{\Omega} W(D \mathbf{y}) d \mathbf{x}+\int_{S_{\mathbf{y}}} g\left(\mathbf{y}^{+}-\mathbf{y}^{-}, \nu_{\mathbf{y}}\right) d \mathcal{H}^{2},
$$


where $\mathbf{y}$ belongs to the space $S B V(\Omega)$ of mappings of special bounded variation, i.e. those whose gradient is a bounded measure having no Cantor part. $S_{\mathbf{y}}$ denotes the set of jump points of $\mathbf{y}, \nu_{\mathbf{y}}$ is the normal to $S_{\mathbf{y}}$ at $\mathbf{y}$, and $\mathbf{y}^{+}, \mathbf{y}^{-}$are the traces of $\mathbf{y}$ from the positive and negative sides of $S_{\mathbf{y}}$ respectively. It is tempting to think of a progression from zero-dimensional (cavitation) to one-dimensional (line singularities) to two-dimensional (cracks) fracture singularities, and there is some evidence that, for example, cavities can coalesce to form cracks. Thus a framework in which all these kinds of singularities can energetically compete is desirable. Recent progress in this direction, leading to a theory in which both cavitation and cracks are possible, has been made by Henao \& Mora-Corral [18; 19].

\subsection{Dynamics}

We end with some brief remarks on dynamics. The balance laws of linear momentum and energy lead to the pointwise forms of the governing equations:

$$
\left.\begin{array}{l}
\rho_{R} \mathbf{y}_{t t}-\operatorname{Div} \mathbf{T}_{R}-\mathbf{b}=0, \\
\left(\frac{1}{2} \rho_{R}\left|\mathbf{y}_{t}\right|^{2}+U\right)_{t}-\mathbf{b} \cdot \mathbf{y}_{t}-\operatorname{Div}\left(\mathbf{y}_{t} \mathbf{T}_{R}\right)+\operatorname{Div} \mathbf{q}_{R}-r=0,
\end{array}\right\}
$$

where $\rho_{R}>0$ is the density in the reference configuration, $\mathbf{b}$ is the body force, $U$ is the internal energy density, and $\mathbf{q}_{R}$ the reference heat flux vector.

The balance of angular momentum holds if and only if the Cauchy stress tensor

$$
\mathbf{T}=(\operatorname{det} D \mathbf{y})^{-1} \mathbf{T}_{R}(D \mathbf{y})^{T}
$$

is symmetric.

For a thermoelastic material, we assume that $\mathbf{T}_{R}$, the entropy density $\eta$, the Helmholtz free energy $\psi=U-\theta \eta$ and $\mathbf{q}_{R}$ depend on $D \mathbf{y}, \theta$, and $\operatorname{Grad} \theta$. Use of the Clausius-Duhem inequality then leads to

$$
\psi=\psi(D \mathbf{y}, \theta), \quad \mathbf{T}_{R}=D_{\mathbf{A}} \psi, \quad \eta=-D_{\theta} \psi
$$

and

$$
-\mathbf{q}_{R} \cdot \operatorname{Grad} \theta \geq 0 .
$$

Frame-indifference is equivalent to

$$
\psi(\mathbf{R A}, \theta)=\psi(\mathbf{A}, \theta) \text { for all } \mathbf{R} \in S O(3),
$$

and this implies that $\mathbf{T}$ is symmetric. We need to solve (5) for the unknowns $\mathbf{y}$ and $\theta$. 
If we assume that $\theta(\mathbf{x}, t)=\theta_{0}=$ constant then we obtain the equation of motion

$$
\rho_{R} \mathbf{y}_{t t}-\operatorname{Div} D_{\mathbf{A}} W(D \mathbf{y})-\mathbf{b}=0,
$$

where $W(\mathbf{A})=\psi\left(\mathbf{A}, \theta_{0}\right)$. This is a multi-dimensional system of conservation laws about which very little is known. One might ask, however, if polyconvexity or quasiconvexity play any role. Whereas nothing seems to be known about their implications for existence of solutions, there are two such results as regards uniqueness:

1. (Dafermos [14]) If $W$ is quasiconvex then Lipschitz solutions of uniformly small oscillation are unique within the class of weak solutions.

2. (Le Floch, Qin [30]) If $W$ is polyconvex, the hypothesis of uniform small oscillation in the Dafermos result can be removed.

What if we add dissipation? The simplest situation is that of viscoelasticity of rate type, for which the equation of motion is

$$
\rho_{R} \mathbf{y}_{t t}-\mathbf{T}_{R}\left(D \mathbf{y}, D \mathbf{y}_{t}\right)=0 .
$$

Frame-indifference of $\mathbf{T}_{R}$ holds if and only if

$$
\mathbf{T}_{R}\left(D \mathbf{y}, D \mathbf{y}_{t}\right)=D \mathbf{y} \mathbf{G}\left(\mathbf{U}, \mathbf{U}_{t}\right),
$$

where $\mathbf{G}$ is symmetric. No large data existence theorem is known for this case (though one would expect to have one that would cover even nonquasiconvex elastic energies). With a good existence and uniqueness theory we could study the questions of approach to equilibrium and address qualitative features of the dynamic evolution.

\section{Acknowledgement}

The author gratefully acknowledges the support of EPSRC through the New Frontiers in the Mathematics of Solids (OxMOS) programme and the Oxford Centre for Nonlinear PDE (OxPDE). The paper was completed while he was enjoying the hospitality of the Laboratoire Jacques-Louis Lions, Paris, supported by the Université Pierre et Marie Curie and CNRS.

\section{Bibliography}

[1] E. Acerbi and N. Fusco. Semicontinuity problems in the calculus of variations. Arch. Rational Mech. Anal., 86:125-145, 1984.

[2] R.A. Adams and J.J.F. Fournier. Sobolev Spaces. Academic Press, Second edition, 2003. 
[3] J.M. Ball. Convexity conditions and existence theorems in nonlinear elasticity. Arch. Rational Mech. Anal., 63:337-403, 1977.

[4] J.M. Ball. Strict convexity, strong ellipticity, and regularity in the calculus of variations. Proc. Camb. Phil. Soc., 87:501-513, 1980.

[5] J.M. Ball. Discontinuous equilibrium solutions and cavitation in nonlinear elasticity. Phil. Trans. Royal Soc. London A, 306:557-611, 1982.

[6] J.M. Ball. Singularities and computation of minimizers for variational problems. In R. DeVore, A. Iserles, and E. Suli, editors, Foundations of Computational Mathematics. Cambridge University Press, 2001.

[7] J.M. Ball. Some open problems in elasticity. In Geometry, Mechanics, and Dynamics, pages 3-59. Springer, New York, 2002.

[8] J.M. Ball, B. Kirchheim, and J. Kristensen. Regularity of quasiconvex envelopes. Calculus of Variations and Partial Differential Equations, 11:333359, 2000.

[9] J.M. Ball and J.E. Marsden. Quasiconvexity at the boundary, positivity of the second variation, and elastic stability. Arch. Rational Mech. Anal., $86: 251-277,1984$.

[10] J.M. Ball and V.J. Mizel. One-dimensional variational problems whose minimizers do not satisfy the Euler-Lagrange equations. Arch. Rational Mech. Anal., 90:325-388, 1985.

[11] J.M. Ball and F. Murat. $W^{1, p}$-quasiconvexity and variational problems for multiple integrals. J. Functional Analysis, 58:225-253, 1984.

[12] J.C. Bellido and C. Mora-Corral. Approximation of Hölder continuous homeomorphisms by piecewise affine homeomorphisms. arXiv:0806.3366v1 [math.CA]

[13] B. Dacorogna. Quasiconvexity and relaxation of non convex variational problems. J. Funct. Anal., 46:102-118, 1982.

[14] C.M. Dafermos. Hyperbolic Conservation Laws in Continuum Physics. Second Edition, volume 325 of Grundlehren der Mathematischen Wissenschaften, Springer-Verlag, Berlin, 2005.

[15] L.C. Evans. Quasiconvexity and partial regularity in the calculus of variations. Arch. Rational Mech. Anal., 95:227-268, 1986.

[16] G.A. Francfort and J.-J. Marigo. Revisiting brittle fracture as an energy minimization problem. J. Mech. Phys. Solids, 46:1319-1342, 1998.

[17] Y. Grabovsky and T. Mengesha. Sufficient conditions for strong local minima: the case of $C^{1}$ extremals. Trans. Amer. Math. Soc., 361:14951541, 2009.

[18] D. Henao and C. Mora-Corral. Invertibility and weak continuity of the determinant for the modelling of cavitation and fracture in nonlinear elasticity, in preparation.

[19] D. Henao and C. Mora-Corral. Fracture surfaces and the regularity of inverses for BV deformations, in preparation. 
[20] B. Kirchheim and J. Kristensen. Differentiability of convex envelopes. C. R. Acad. Sci. Paris Sér. I Math., 333:725-728, 2001.

[21] J.K. Knowles and E. Sternberg. On the failure of ellipticity and the emergence of discontinuous deformation gradients in plane finite elastostatics. J. Elasticity, 8:329-379, 1978.

[22] J. Kristensen. On the non-locality of quasiconvexity. Ann. Inst. H. Poincaré, Anal. Non Linéaire, 16:1-13, 1999.

[23] J. Kristensen and G. Mingione. The singular set of Lipschitzian minima of multiple integrals. Arch. Rational Mech. Anal., 184:341-369, 2007.

[24] J. Kristensen and A. Taheri. Partial regularity of strong local minimizers in the multi-dimensional calculus of variations. Arch. Rational Mech. Anal., 170:63-89,2003.

[25] C. Mora-Corral. Approximation by piecewise homeomorphisms of Sobolev homeomorphisms that are smooth outside a point. Houston J. Math., in press.

[26] C.B. Morrey. Quasi-convexity and the lower semicontinuity of multiple integrals. Pacific J. Math., 2:25-53, 1952.

[27] C.B. Morrey. Multiple Integrals in the Calculus of Variations, Springer, 1966.

[28] S. Müller, T. Qi, and B.S. Yan. On a new class of elastic deformations not allowing for cavitation. Ann. Inst. Henri Poincaré, Analyse Nonlinéaire, 11:217-243, 1994.

[29] J. Nečas. Example of an irregular solution to a nonlinear elliptic system with analytic coefficients and conditions for regularity. In Theory of Nonlinear Operators, pages 197-206, Berlin, 1977. Akademie-Verlag.

[30] T. Qin. Symmetrizing the nonlinear elastodynamic system. J. Elasticity, 50:245-252, 1998.

[31] E.N. Spadaro. Non-uniqueness of minimizers for strictly polyconvex functionals. Arch. Rational Mech. Anal., 2009, in press.

[32] V. Šverák. Quasiconvex functions with subquadratic growth. Proc. Roy. Soc. Lond. A, 433:723-732, 1991.

[33] V. Šverák and X. Yan. A singular minimizer of a smooth strongly convex functional in three dimensions. Calc. Var. Partial Differential Equations, 10:213-221, 2000.

[34] C. Truesdell and W. Noll, The non-linear field theories of mechanics. In Handbuch der Physik, Vol. III/3m ed. S. Flügge, Springer, Berlin, 1965. 